\title{
A Symmetric Two Higgs Doublet Model
}

\author{
Hannah Bossi and Shreyashi Chakdar
}

\section{Journal of Nepal Physical Society}

Volume 7, No 3, 2021

(Special Issue: ANPA Conference, 2021)

ISSN: 2392-473X (Print), 2738-9537 (Online)

\section{Editors:}

Dr. Nabin Malakar (Editor in chief)

Worcester State University

Dr. Pashupati Dhakal

Thomas Jefferson National Accelerator Facility, USA

Dr. Arjun Dahal

University of South Alabama, USA

Dr. Chiranjivi Lamsal

SUNY Platsburgh, USA

Dr. Dilli Raj Paudyal

University of Regina, Canada

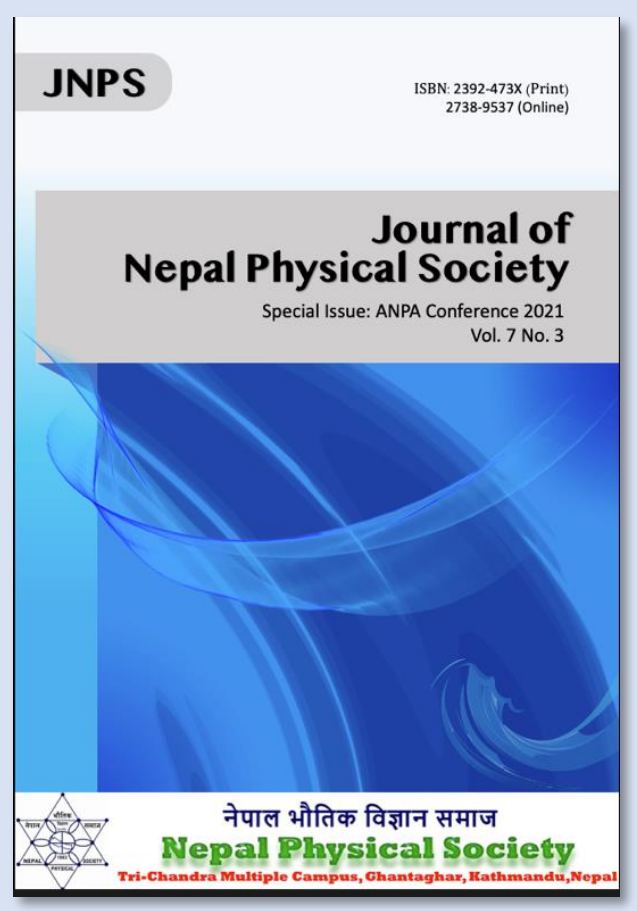

\section{Managing Editor:}

Dr. Binod Adhikari

St. Xavier's College, Kathmandu, Nepal

JNPS, 7 (3), 34-40 (2021)

DOI: http://doi.org/10.3126/jnphyssoc.v7i3.42189

\section{Published by: Nepal Physical Society}

P.O. Box: 2934

Tri-Chandra Campus

Kathmandu, Nepal

Email: npseditor@gmail.com 


\title{
A Symmetric Two Higgs Doublet Model
}

\author{
Hannah Bossi ${ }^{1}$ and Shreyashi Chakdar ${ }^{2}$ \\ 1) Department of Physics, Yale University, New Haven, CT 06511,USA ${ }^{\text {a) }}$ \\ ${ }^{2)}$ Department of Physics, College of the Holy Cross, Worcester, MA 01609, USA. ${ }^{\text {b) }}$ \\ a) Electronic mail: hannah.bossi@yale.edu

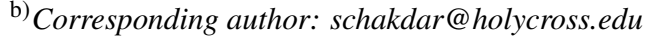

\begin{abstract}
In the light of ongoing experimental search efforts for the dark matter and the post-Higgs Beyond the Standard Model (BSM) null results at the Large Hadron Collider (LHC), the Electroweak sector demands to be investigated for possible new scalar states discoverable at the LHC fulfilling the role of the dark matter. In this work we present a symmetric two Higgs doublet model with a discrete interchange symmetry among the two Higgs doublets $\left(\Phi_{1} \leftrightarrow \Phi_{2}\right)$. Apart from the Standard Model (SM)-like scalar state (h) with $m_{h}=125 \mathrm{GeV}$, the model has several distinguishing features including the pseudoscalar (A), the charged scalars $\left(H^{ \pm}\right)$ and the neutral scalar $\mathrm{H}$, not having any direct coupling to the fermions. The neutral scalar $H$ is assumed to have mass lighter than the $125 \mathrm{GeV}$ SM-like Higgs state $h$. Due to the presence of a residual $Z_{2}$ symmetry after the spontaneous symmetry breaking (SSB), the neutral scalar $H$ can emerge as a viable dark matter candidate. We discuss the constraints on such scalar dark matter from the current direct and indirect detection experiments. As a by-product of this construction, the SM-like scalar $h$ ends up having an extra invisible decay mode of $h \rightarrow H H$ in this model which can also influence the dark matter parameter space. We discuss these model features in detail along with a guideline of relevant phenomenological searches at the LHC for this scenario.
\end{abstract}

Received: 14 August 2021; Revised: 30 October 2021; Accepted: 15 November 2021

Keywords: Higgs doublets, Dark Matter, LHC

\section{INTRODUCTION}

With the discovery of a Higgs-like particle $h$ with mass $m_{h}$ around $125 \mathrm{GeV}$ by the ATLAS and CMS experiments at the LHC [1, 2], an era of exploration in the Electroweak sector had begun. With only this stand-alone SM-like scalar state being observed and ongoing experimental searches for dark matter, it is even more vital now to explore the scalar sector of different BSM scenarios in utmost detail to investigate the possibility of a spectrum of additional scalar states fulfilling the role of the dark matter. In this scenario, the simplest and well motivated BSM extension to be considered are the TwoHiggs-Doublet Models (2HDM's) [3], in which adding a second $S U(2)_{L}$ higgs doublet leads to five physical scalar particles: two neutral scalars $h, H$, pseudoscalar $A$ and charged-scalar $H^{ \pm}$. Many versions of the 2HDMs have been extensively studied in the past [4]. Some of the notables include: (a) Supersymmetric two Higgs doublet model [5], (b) Non-supersymmetric two Higgs doublet models: (i) with only one Higgs doublet (conventionally chosen to be $\Phi_{2}$ ) with couplings to the fermions (type I 2HDM), (ii) with both Higgs doublets having VEVs, additionally with one doublet (conventionally chosen to be $\Phi_{2}$ ) coupling to the up type quarks only, while the other $\left(\Phi_{1}\right)$ coupling to the down type quarks only (type II $2 \mathrm{HDM}$ ),(iii) with one $\operatorname{doublet}\left(\Phi_{2}\right)$ coupling to the up type quarks only and the other $\left(\Phi_{1}\right)$ coupling to the down type quarks only i.e just like type II $2 \mathrm{HDM}$ along with RH leptons coupling to $\Phi_{2}$ ("flipped" model or type Y), (iv) with RH quarks coupling to one Higgs doublet $\left(\Phi_{2}\right)$ and RH leptons coupling to another Higgs $\operatorname{doublet}\left(\Phi_{1}\right)$ ("lepton-specific" or type X) and (v) with only one doublet with VEVs and couplings to the fermions("Inert Doublet Model"(IDM)) [6]. Different variations of all these models have attracted a lot of attention recently and were investigated in view of the recent LHC data $[7,8,9,10$, $11,12,13,14,15,16,17,18,19,20,21]$ In this work, we present a symmetric two Higgs doublet model with a discrete interchange symmetry $\left(\Phi_{1} \leftrightarrow \Phi_{2}\right)$ with several distinguishing features:

(i) The neutral scalar $h$ represents the Standard Model(SM)like Higgs with mass $m_{h} \simeq 125 \mathrm{GeV}$.

(ii) No flavor-changing neutral currents (FCNC) is present. (iii) The other neutral scalar $H$ can be lighter than the SM-like neutral scalar state $h$ and couples only to the Electroweak Gauge Bosons $\left(W^{ \pm}\right.$and $Z$ ) but does not couple to fermions at all. 
(iv) The charged Higgs $H^{ \pm}$and the pseudoscalar $A$ don't couple to the fermions. $H^{ \pm}$only couples to the gauge bosons, the neutral scalar $H$ and pseudoscalar $A$.

(v) The pseudoscalar only couples to the gauge bosons and the charged scalar $H^{ \pm}$but does not couple to either the lighter and heavier neutral scalars $(H$ and $h)$.

(vi) At the LHC, the lighter scalar $H$ can only be produced via the decays of the SM-like neutral scalar $h$. As the lighter scalar $H$ does not couple to the fermions at all, the mass limit on $H$ is significantly lower than the mass limits coming from gluon-gluon fusion LHC data for production of a generic scalar boson.

(vii) The charged Higgs $H^{ \pm}$can be produced via the Drell-Yan process. It will be quite elusive to discover it at the LHC with the decay products of " $2 \mathrm{~W}$-boson + Miss $E_{T}$ " in the final state.

(viii) After the $\Phi_{1} \leftrightarrow \Phi_{2}$ interchange symmetry is spontaneously broken, there is a residual $Z_{2}$ symmetry that remains unbroken making the scalar states $H^{ \pm}, H$ and $A$ $Z_{2}$ negative, while all other fields $Z_{2}$ positive. Thus the lightest $Z_{2}$ negative particle (either $H$ or $A$ ) will be a candidate for the dark matter in this scenario.

(ix) If the lightest scalar $H$ is considered as the DM, the decay channel $h \rightarrow H H$ will act as an extra invisible decay mode for the SM-like Higgs $\left(m_{h} \simeq 125 \mathrm{GeV}\right)$ and a bound in the parameter space of the effective coupling of $h$ and $H$ and the mass of the lightest scalar $m_{H}$ can be obtained from the invisible Higgs branching ratio bound for the SM-like state: $B R_{i n v h}<26 \%$.

(x) We also investigate the various bounds on the dark matter parameter space; for example, bounds from Electroweak precision constraints and direct and Indirect detection are discussed.

(xi) The neutral Higgs $H$ has a coupling $H H Z Z$ which can lead to interesting decay process $Z \rightarrow Z^{*} H H \rightarrow f \bar{f} H H$ and can be tested at the proposed $e^{+} e^{-}$collider ILC. [22] Below we present the Model and formalism, followed by the phenomenological implications for this "Symmetric two Higgs doublet model."

\section{THE MODEL AND FORMALISM}

Our proposed model is based on the Standard Model $(\mathrm{SM})$ symmetry group $S U(3)_{C} \times S U(2)_{L} \times U(1)_{Y}$ with the Higgs sector being extended minimally with two Higgs doublets $\Phi_{1}$ and $\Phi_{2}$. We consider a discrete interchange symmetry of $\Phi_{1} \leftrightarrow \Phi_{2}$ with the condition of $V_{\Phi_{1}}=V_{\Phi_{2}}$. Thus the proposed framework is named "A symmetric two Higgs doublet model".

The vacuum expectation value's (VEV) of these Higgs doublets are summarized below:

$$
\left\langle\Phi_{1}\right\rangle=\frac{1}{\sqrt{2}}\left(\begin{array}{c}
0 \\
V_{\Phi_{1}}
\end{array}\right),\left\langle\Phi_{2}\right\rangle=\frac{1}{\sqrt{2}}\left(\begin{array}{c}
0 \\
V_{\Phi_{2}}
\end{array}\right) .
$$

In the unitary gauge, the two doublets can be written as,

$$
\Phi_{1}=\frac{1}{\sqrt{2}}\left(\begin{array}{c}
\frac{\sqrt{2} V_{\Phi_{2}}}{V} H^{+} \\
h_{0}+i \frac{V_{\Phi_{2}}}{V} A+V_{\Phi_{1}}
\end{array}\right)
$$

$$
\Phi_{2}=\frac{1}{\sqrt{2}}\left(\begin{array}{c}
\frac{-\sqrt{2} V_{\Phi_{1}}}{V} H^{+} \\
H_{0}-i \frac{V_{\Phi_{1}}}{V} A+V_{\Phi_{2}}
\end{array}\right)
$$

where $V_{\Phi_{1}}=V_{\Phi_{2}}=v / \sqrt{2}$ and $v^{2}=V_{\Phi_{1}}^{2}+V_{\Phi_{1}}^{2}=(250)^{2}$ $\mathrm{GeV}$ where $v$ is the vacuum expectation value (VEV) for the Higgs field. The five physical scalar fields are $h, H, A$ and $H^{ \pm}$which are respectively the two neutral scalars, the psuedoscalar and the charged scalar. After the $\Phi_{1} \leftrightarrow$ $\Phi_{2}$ interchange symmetry is spontaneously broken, there is a residual $Z_{2}$ symmetry that remains unbroken. This residual symmetry makes $H^{ \pm}, H$ and $A$ acquire $Z_{2}$ negative charges i.e $H^{ \pm} \rightarrow-H^{ \pm} ; H \rightarrow-H ; A \rightarrow-A$ while all other fields are $Z_{2}$ positive. As a result, the lightest $Z_{2}$ negative particle (either $H$ or $A$ ) can be a suitable dark matter candidate. The most general potential with this exchange symmetry of $\Phi_{1} \leftrightarrow \Phi_{2}$ can be written as,

$$
\begin{aligned}
V= & +m_{11}^{2} \Phi_{1}^{\dagger} \Phi_{1}+m_{22}^{2} \Phi_{2}^{\dagger} \Phi_{2} \\
& -m_{12}^{2} \Phi_{1}^{\dagger} \Phi_{2}-m_{12}^{* 2} \Phi_{2}^{\dagger} \Phi_{1}+\frac{\lambda_{1}}{2}\left(\Phi_{1}^{\dagger} \Phi_{1}\right)^{2} \\
& +\frac{\lambda_{2}}{2}\left(\Phi_{2}^{\dagger} \Phi_{2}\right)^{2}+\lambda_{3}\left(\Phi_{1}^{\dagger} \Phi_{1}\right)\left(\Phi_{2}^{\dagger} \Phi_{2}\right) \\
& +\lambda_{4}\left(\Phi_{1}^{\dagger} \Phi_{2}\right)\left(\Phi_{2}^{\dagger} \Phi_{1}\right) \\
& +\frac{\lambda_{5}}{2}\left(\Phi_{1}^{\dagger} \Phi_{2}\right)^{2}+\frac{\lambda_{5}^{*}}{2}\left(\Phi_{2}^{\dagger} \Phi_{1}\right)^{2} \\
& +\lambda_{6}\left(\Phi_{1}^{\dagger} \Phi_{1}\right)\left(\Phi_{1}^{\dagger} \Phi_{2}\right) \\
& +\lambda_{6}^{*}\left(\Phi_{1}^{\dagger} \Phi_{1}\right)\left(\Phi_{2}^{\dagger} \Phi_{1}\right) \\
& +\lambda_{7}\left(\Phi_{2}^{\dagger} \Phi_{2}\right)\left(\Phi_{1}^{\dagger} \Phi_{2}\right) \\
& +\lambda_{7}^{*}\left(\Phi_{2}^{\dagger} \Phi_{2}\right)\left(\Phi_{2}^{\dagger} \Phi_{1}\right)
\end{aligned}
$$

where due to the exchange symmetry of $\Phi_{1} \leftrightarrow \Phi_{2}$, we take,

$$
m_{11}^{2}=m_{22}^{2}, m_{12}^{2}=m_{12}^{* 2}, \lambda_{1}=\lambda_{2}, \lambda_{5}=\lambda_{5}^{*}, \lambda_{6}=\lambda_{7}^{*}
$$

so that we have total 8 parameters in the potential. Minimization of this potential gives us two solutions,

$$
\begin{gathered}
V_{\Phi_{1}}=V_{\Phi_{2}}, \\
m_{11}^{2}+m_{12}^{2}+ \\
\lambda_{1}\left(V_{\Phi_{1}}^{2}+V_{\Phi_{2}}^{2}+V_{\Phi_{1}} V_{\Phi_{2}}\right)- \\
\left(\lambda_{3}+\lambda_{4}+\lambda_{5}\right) V_{\Phi_{1}} V_{\Phi_{2}}- \\
\lambda_{6}\left(V_{\Phi_{1}}^{2}+V_{\Phi_{2}}^{2}\right)+2 \lambda_{6}^{*} V_{\Phi_{1}} V_{\Phi_{2}}=0
\end{gathered}
$$

among which we choose the first solution of minimization i.e $V_{\Phi_{1}}=V_{\Phi_{2}}=v / \sqrt{2}=175 \mathrm{GeV}$. From the determination of the second derivatives of the potential $\mathrm{V}$ referred in Eqn(4), the first minimization solution of Eqn(5) can be taken as a local minima along with the following condition,

$$
\left(\lambda_{1}+\lambda_{5}+2\left[\lambda_{6}^{*}\right]\right) \geq 0
$$

In this work, we consider the first minimization solution with $V_{\Phi_{1}}=V_{\Phi_{2}}=v / \sqrt{2}=175 \mathrm{GeV}$ along with the condition stated in Eqn(6) above. With the above minimum, the particle masses are given by, 


$$
\begin{array}{r}
m_{w}^{2}=\frac{g^{2} v^{2}}{4}, m_{Z}^{2}=\frac{\left(g^{2}+g^{2}\right) v^{2}}{4}, \\
m_{h}^{2}=\left(\lambda_{3}+\lambda_{4}+\lambda_{5}-\lambda_{1}\right) v^{2} / 2, \\
m_{H}^{2}=2 m_{12}^{2}-\left[\lambda_{1}+\lambda_{3}+\lambda_{4}+\lambda_{5}+2\left(\lambda_{6}+\lambda_{6}^{*}\right)\right] v^{2} / 2, \\
m_{A}^{2}=2 m_{12}^{2}-\left[\lambda_{5}+\lambda_{6}+\lambda_{6}^{*}\right] v^{2}, \\
m_{H^{ \pm}}^{2}=2 m_{12}^{2}-\left[\lambda_{4}+\lambda_{5}+\lambda_{6}+\lambda_{6}^{*}\right] v^{2},
\end{array}
$$

where the two neutral scalars $h$ and $H$ are defined as,

$$
\begin{array}{r}
h=h^{0} \cos (\alpha)+H^{0} \sin (\alpha), \\
H=-h^{0} \sin (\alpha)+H^{0} \cos (\alpha)
\end{array}
$$

where $\alpha=$ the scalar diagonalising angle $=\pi / 4$. Also, here $\tan \beta=V_{\Phi_{2}} / V_{\Phi_{1}}=1$.

From the assumption that we take the neutral scalar $H$ to be lighter than the SM-like neutral scalar $h$ and $m_{H}^{2}<$ $m_{h}^{2}$, we get a condition on the linear combination of the parameters as below

$$
m_{12}^{2}<\left[\lambda_{3}+\lambda_{4}+\lambda_{5}+\lambda_{6}+\lambda_{6}^{*}\right] v^{2} / 2
$$

Now as the neutral scalar $h$ is considered as the SM Higgslike state with $m_{h} \simeq 125 \mathrm{GeV}$, we get another condition on the relevant parameters given by

$$
\lambda_{3}+\lambda_{4}+\lambda_{5}-\lambda_{1}=0.5
$$

Also, the potential must be bounded from below and the corresponding vacuum stability conditions are given by,

$$
\begin{array}{r}
\sqrt{4 \pi}>\lambda_{1} \geq 0, \sqrt{4 \pi}>\lambda_{2} \geq 0, \\
\lambda_{3} \geq-\sqrt{\lambda_{1} \lambda_{2}}, \lambda_{3}+\lambda_{4}-\left[\lambda_{5}\right] \geq-\sqrt{\lambda_{1} \lambda_{2}},
\end{array}
$$

Note that these conditions are only valid for $\lambda_{6}=\lambda_{7}=0$.

Furthermore, from the Large Electron-Positron Collider (LEP) experiment we have constraints on the Z boson decay width and mass of the charged scalar $\left(H^{ \pm}\right)$ [23] given by,

$$
\begin{aligned}
& m_{H}+m_{A}>m_{Z}, \\
& m_{H}^{ \pm}>79.3 \mathrm{GeV} .
\end{aligned}
$$

If we now look at the Yukawa interaction Lagrangian relevant under our gauge symmetry as well as the discrete interchange symmetry of $\Phi_{1} \leftrightarrow \Phi_{2}$ given by,

$$
\begin{aligned}
\mathscr{L} & \supset f_{d}^{\Phi_{1}}\left(\overline{u_{L}}, \bar{d}_{L}\right) d_{R} \Phi_{1} \\
& +f_{u}^{\Phi_{1}}\left(\overline{u_{L}}, \bar{d}_{L}\right) u_{R} \tilde{\Phi}_{1} \\
& +f_{d}^{\Phi_{2}}\left(\overline{u_{L}}, \bar{d}_{L}\right) d_{R} \Phi_{2}+f_{u}^{\Phi_{2}}\left(\overline{u_{L}}, \bar{d}_{L}\right) u_{R} \tilde{\Phi_{2}},
\end{aligned}
$$

where because of the interchange symmetry $\Phi_{1} \leftrightarrow \Phi_{2}$, we take

$$
f_{d}^{\Phi_{1}}=f_{d}^{\Phi_{2}}=f_{d}, f_{u}^{\Phi_{1}}=f_{u}^{\Phi_{2}}=f_{u}
$$

from this we can obtain the charged scalar $\left(H^{ \pm}\right)$couplings, the neutral scalar $(h, H)$ couplings and the pseudoscalar $(A)$ couplings with the fermions. We find that only the neutral scalar $(h)$ couple to the fermions with the couplings of $f_{u}$ and $f_{d}$ (same as in the SM) and the other scalars $\left(H, H^{ \pm}, A\right)$ do not couple to the fermions at all. Again, looking at the gauge bosons masses and mixings are obtained from the kinetic terms of the scalars in the Lagrangian, we get

$$
\mathscr{L} \supset\left(\mathscr{D}_{\mu} \Phi_{1}\right)^{\dagger}\left(\mathscr{D}^{\mu} \Phi_{1}\right)+\left(\mathscr{D}_{\mu} \Phi_{2}\right)^{\dagger}\left(\mathscr{D}^{\mu} \Phi_{2}\right),
$$

where, $\mathscr{D}$ is the covariant derivative associated with the gauge group, given by

$$
\mathscr{D}_{\mu}=\partial_{\mu}-i g \frac{\tau_{a}}{2} A_{\mu}^{a}-i g^{\prime} \frac{Y}{2} B_{\mu},
$$

where $\tau_{a}$ 's are the Pauli matrices. The relevant interactions of the scalar fields $h^{0}, H^{0}, H^{ \pm}$and $A$ with the gauge bosons ( $W^{ \pm}$and $\mathrm{Z}$ ) before the spontaneous symmetry breaking are given by,

$$
\begin{aligned}
\mathscr{L}_{\text {gauge }} & \supset \mathrm{gm}_{w} W_{\mu}^{+} W_{\mu}^{-} \frac{h^{0}+H^{0}}{\sqrt{2}} \\
& +\frac{m_{Z}^{2}}{v} Z_{\mu} Z_{\mu} \frac{h^{0}+H^{0}}{\sqrt{2}} \\
& +\frac{m_{w}^{2}}{v^{2}} W_{\mu}^{+} W_{\mu}^{-}\left(\left(h^{0}\right)^{2}+\left(H^{0}\right)^{2}\right) \\
& +\frac{m_{Z}^{2}}{2 v^{2}} Z_{\mu} Z_{\mu}\left(\left(h^{0}\right)^{2}+\left(H^{0}\right)^{2}\right) \\
& -\frac{g}{2}\left(\partial_{\mu} H^{ \pm}\right) W_{\mu}^{ \pm} \frac{H^{0}-h^{0}}{\sqrt{2}} \\
& -\frac{g}{2} \partial_{\mu}\left(\frac{H^{0}-h^{0}}{\sqrt{2}}\right) W_{\mu}^{ \pm} H^{ \pm} \\
& \frac{i g}{2}\left(\partial_{\mu} H^{\mp}\right) W_{\mu}^{ \pm} A \pm \frac{i g}{2}\left(\partial_{\mu} A\right) W_{\mu}^{ \pm} H^{\mp}
\end{aligned}
$$

Now with the definition of the mass eigenstates $h$ and $H$ as stated in eqn(8) with $\alpha=$ the scalar diagonalizing angle $=\pi / 4$ we get,

$$
h=\left(h^{0}+H^{0}\right) / \sqrt{2}, H=\left(-h^{0}+H^{0}\right) / \sqrt{2}
$$

Using these definitions in Eqn(16) we get the relevant interactions after the SSB as,

$$
\begin{aligned}
\mathscr{L}_{\text {gauge }} & \supset g m_{w} W_{\mu}^{+} W_{\mu}^{-} h+\frac{m_{Z}^{2}}{v} Z_{\mu} Z_{\mu} h \\
& +\frac{m_{w}^{2}}{v^{2}} W_{\mu}^{+} W_{\mu}^{-} h^{2}+\frac{m_{w}^{2}}{v^{2}} W_{\mu}^{+} W_{\mu}^{-} H^{2} \\
& +\frac{m_{Z}^{2}}{2 v^{2}} Z_{\mu} Z_{\mu} h^{2}+\frac{m_{Z}^{2}}{2 v^{2}} Z_{\mu} Z_{\mu} H^{2} \\
& -\frac{g}{2}\left(\partial_{\mu} H^{ \pm}\right) W_{\mu}^{ \pm} H-\frac{g}{2}\left(\partial_{\mu} H\right) W_{\mu}^{ \pm} H^{ \pm} \\
& \pm \frac{i g}{2}\left(\partial_{\mu} H^{\mp}\right) W_{\mu}^{ \pm} A \\
& \pm \frac{i g}{2}\left(\partial_{\mu} A\right) W_{\mu}^{ \pm} H^{\mp}
\end{aligned}
$$

Interestingly, as seen from the above interactions, only the SM-like scalar $h$ has three point couplings with the gauge bosons $\left(W^{ \pm}\right.$and $\mathrm{Z}$ ) same with the couplings found in the Standard Model (SM), where as the other neutral scalar $H$ does not have any three point couplings of this sort. However, the neutral scalar $H$ has four point couplings with the gauge bosons $\left(W_{\mu}^{+} W_{\mu}^{-} H H\right.$ and $\left.Z_{\mu} Z_{\mu} H H\right)$. The charged scalars $\left(H^{ \pm}\right)$couple to both the neutral Higgs $H$ and the pseudoscalar $A$ along with the gauge boson $W^{ \pm}$ whereas the pseudoscalar $A$ does not couple to the neutral scalar state $H$ but only couples to the charged scalar $\left(H^{ \pm}\right)$ and the gauge boson $W^{ \pm}$. 
From the Higgs potential, we get these relevant interactions of the scalar fields $h^{0}, H^{0}, A$ and $H^{ \pm}$before the SSB,

$$
\begin{aligned}
\mathscr{L}_{\text {scalar }} & \supset \frac{\left(\lambda_{3}+\lambda_{4}+\lambda_{5}\right)}{4}\left(\left(h^{0}\right)^{2}\left(H^{0}\right)^{2}\right. \\
& \left.+\sqrt{2} v\left(\left(h^{0}\right)^{2} H^{0}+\left(H^{0}\right)^{2} h^{0}\right)\right) \\
& +\frac{\left(\lambda_{6}+\lambda_{6}^{*}\right)}{4}\left(\left(\left(h^{0}\right)^{3} H^{0}+\left(H^{0}\right)^{3} h^{0}\right)\right. \\
& \left.+\frac{3 v\left(\left(h^{0}\right)^{2} H^{0}+\left(H^{0}\right)^{2} h^{0}\right)}{\sqrt{2}}\right)
\end{aligned}
$$

After the SSB the relevant interactions between the two neutral scalars $h$ and $H$ are given by,

$$
\begin{aligned}
\mathscr{L}_{\text {scalar }} & \supset-\frac{\left(\lambda_{3}+\lambda_{4}+\lambda_{5}\right)}{4} h^{2} H^{2} \\
& -\left(\frac{2\left(\lambda_{3}+\lambda_{4}+\lambda_{5}\right)+3\left(\lambda_{6}+\lambda_{6}^{*}\right)}{8}\right) v h H^{2}
\end{aligned}
$$

So it can be seen from the interactions that there is a four point coupling $h h H H$ and a three point coupling $h H H$ between the two neutral scalars $h$ and $H$.

\section{DARK MATTER CANDIDATE}

As discussed before, after the $\Phi_{1} \leftrightarrow \Phi_{2}$ interchange symmetry is spontaneously broken, there is a residual $Z_{2}$ symmetry that remains unbroken, as a result of which $H^{ \pm}, H$ and $A$ to acquire $Z_{2}$ negative charges i.e $H^{ \pm} \rightarrow-H^{ \pm}$; $H \rightarrow-H ; A \rightarrow-A$, while all other fields acquire $Z_{2}$ positive charges. Thus the lightest $Z_{2}$ negative particle (either the neutral scalar $H$ or the pseudoscalar $A$ ) can be considered as a possible dark matter candidate in this framework.

In this model the masses of the neutral scalar $H$ and the pseudoscalar $A$ are given by,

$m_{H}^{2}=2 m_{12}^{2}-\left[\lambda_{1}+\lambda_{3}+\lambda_{4}+\lambda_{5}+2\left(\lambda_{6}+\lambda_{6}^{*}\right)\right] v^{2} / 2$, $m_{A}^{2}=2 m_{12}^{2}-\left[\lambda_{5}+\lambda_{6}+\lambda_{6}^{*}\right] v^{2}$,

Assuming $m_{H}^{2}<m_{A}^{2}$ and using the condition referred in Eqn(10) and using the bound on $\lambda_{1}$ stated in Eqn(11), we get a bound on $\lambda_{5}$ for the lighter neutral scalar $H$ to become the lightest $Z_{2}$ negative particle and fulfill the role of the dark matter candidate in this model:

$$
3.67>\lambda_{5} \geq 0.125
$$

Hence the neutral scalar $H$ can serve as the dark matter as it will be the lightest scalar particle protected by the residual $Z_{2}$ symmetry. The couplings of $\mathrm{H}$ relevant for the dark matter interactions are given by,

$$
\begin{aligned}
\mathscr{L}_{\text {scalar }} & \supset \frac{m_{w}^{2}}{v^{2}} W_{\mu}^{+} W_{\mu}^{-} H^{2}+\frac{m_{Z}^{2}}{2 v^{2}} Z_{\mu} Z_{\mu} H^{2} \\
& -\frac{\left(\lambda_{3}+\lambda_{4}+\lambda_{5}\right)}{4} h^{2} H^{2}
\end{aligned}
$$

where the first two terms arise from gauge interaction and the last term comes from the scalar potential. We see from the interactions in eqn(22) that dark matter is able

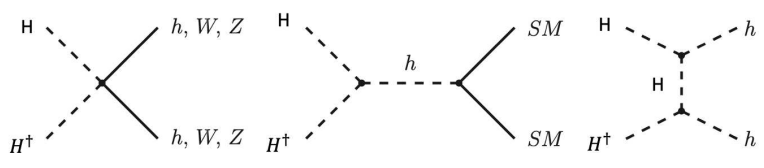

FIGURE 1. Dark matter annihilation channels

to annihilate directly into a pair of $\mathrm{W}, \mathrm{Z}$ or $\mathrm{h}$ if these channels are kinematically open. It can also annihilate to a pair of SM particles through the Higgs boson channel $h$. The annihilation channels are shown in figure and are critical for determining the dark matter relie density as well as possible gamma ray signals from the regions of dark matter population such as dwarf galaxy or from the galactic center. The viable dark matter $H$ must satisfy the correct relic abundance of dark matter obtained by the PLANCK/WMAP collaboration [24] given by,

$$
\Omega_{\text {darkmatter }} h^{2}=0.1199 \pm 0.0027,
$$

where $\Omega$ is the density parameter and $h$ is the Hubble parameter in the unit of $100 \mathrm{~km} \mathrm{~s}^{-1} \mathrm{Mpc}^{-1}$. The dark matter should also satisfy the Electroweak precision constraint bounds [25], dark matter direct detection [26] and indirect detection [27] bounds. As seen from eqn(22), the interaction between a single Higgs boson $h$ and a pair of dark matter is proportional to $\lambda_{345}=\lambda_{3}+\lambda_{4}+\lambda_{5}$, gives rise to dark matter nucleon elastic scattering. In the particular case when $2 m_{H} \leq m_{h}$, the same coupling also lead to decay of the Higgs into a pair of dark matter, as an invisible decay channel of the Higgs boson.

\section{PHENOMENOLOGICAL IMPLICATIONS}

There are several interesting phenomenological implications which can be tested in the next runs of LHC at 14 $\mathrm{TeV}$ and the proposed $e^{+} e^{-}$collider ILC [22]. As the neutral scalar boson $h$ has the same couplings as the SMlike Higgs boson, the production and decays of $h$ will be same as of the SM-like Higgs state with $m_{h} \simeq 125 \mathrm{GeV}$ as seen by the LHC. Interestingly, all the other scalars $\left(H, H^{ \pm}, A\right)$ do not couple to the fermions. For the other neutral scalar $H$, assuming that its mass is lighter that the SM-like Higgs boson mass $\left(m_{H}<m_{h}\right)$, it can be produced via the decays of the SM-like Higgs $h(125 \mathrm{GeV})$, through the three point coupling $h H H$ between $h$ and $H$, as seen in equation(20). As stated previously, as the neutral scalar $H$ is the lightest stable $Z_{2}$ negative particle in the scalar particle mass spectrum, it can not decay to anything further. So the decay of the $125 \mathrm{GeV} h$ into two lighter scalars $H$ will be detected as an extra invisible decay mode for the SM-like scalar $h$ via the decay mode $h \rightarrow H H$ with invisible decay branching ratio as large as $B r_{i n v}<26 \%$ [28]. The expression for the partial decay width of the SM-like 


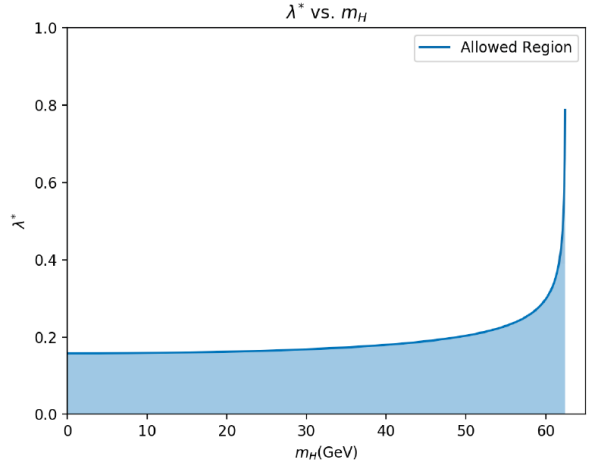

FIGURE 2. Variation of the effective coupling $\lambda^{*}$ vs the DM $\operatorname{mass}\left(m_{H}\right)$ is shown in this figure. The shaded region corresponds to the allowed region in the parameter space for $\lambda^{*}$ and $m_{H}$ coming from the current bound for the SM-like $125 \mathrm{GeV}$ Higgs invisible decay Branching Ratio $<26 \%$.

Higgs boson $h$ with mass of $m_{h} \simeq 125 \mathrm{GeV}$ to two lighter neutral scalar particles $H$ is given by,

$$
\begin{aligned}
\Gamma(h \rightarrow H H) & =\frac{v^{2}}{2048 \pi M_{h}} \\
& *\left(2\left(\lambda_{3}+\lambda_{4}+\lambda_{5}\right)+3\left(\lambda_{6}+\lambda_{6}^{*}\right)\right)^{2} \\
& *\left(1-\frac{4 m_{H}^{2}}{m_{h}^{2}}\right)^{1 / 2}
\end{aligned}
$$

Note that the total width of the SM-like Higgs boson with mass of $125 \mathrm{GeV}$ can be expressed in our model as,

$$
\Gamma_{h}^{t o t}=\sum \Gamma_{h \rightarrow A \bar{A}}+\Gamma_{h \rightarrow H H}
$$

where $A \bar{A}=b \bar{b}, \tau \tau, g g, W W^{*}, Z Z^{*}, c \bar{c}, \gamma \gamma$ and $\sum \Gamma_{h \rightarrow A \bar{A}}$ is taken to be the total decay width of a $125 \mathrm{GeV} \mathrm{SM}$-like Higgs boson $=4.088 \mathrm{MeV}$ [29]. The partial decay width of $\Gamma(h \rightarrow H H)$ adds to the invisible decay branching ratio of the $125 \mathrm{GeV}$ SM-like Higgs. So from the upper bound of $B r_{i n v h}<26 \%$ [28] for the invisible deacy Branching Ratio of the SM-like Higgs particle, we get the following bound on the decay width $(\Gamma(h \rightarrow H H))$

$$
\Gamma(h \rightarrow H H)<1.2775 \times 10^{-3} \mathrm{GeV}
$$

Using Eqn(24) this can be translated as a bound on the effective coupling $\lambda^{*}$ and $m_{H}$ as,

$$
\lambda^{* 2}\left(1-\frac{4 m_{H}^{2}}{m_{h}^{2}}\right)^{1 / 2}<0.01678
$$

where $\lambda^{*}=2\left(\lambda_{3}+\lambda_{4}+\lambda_{5}\right)+3\left(\lambda_{6}+\lambda_{6}^{*}\right)$ is defined to be the effective coupling between $h H H$. The variation of $\lambda^{*}$ vs $m_{H}$ is shown in $\operatorname{Fig}(2)$. The shaded region corresponds to the allowed parameter space from the invisible decay branching ratio bound of $B r_{i n v h}<26 \%$ for the 125 $\mathrm{GeV}$ Higgs boson. It can be seen in Fig(2) that the effective coupling $\lambda^{*}$ seems to be varying very slowly with $m_{H}$ for the lower neutral scalar mass $\left(m_{H}\right)$ values while at $m_{H}=62.5$, the effective coupling $\lambda^{*}$ becomes infinity.
Note that the bounds which are reflected in Figure 2 only reflect to the invisible Higgs decay LHC bounds. In order to study the parameter space in detail, one needs to consider direct and indirect detection along with Electroweak precision constraint bounds.

To consider the effects coming from the Electroweak Pre-

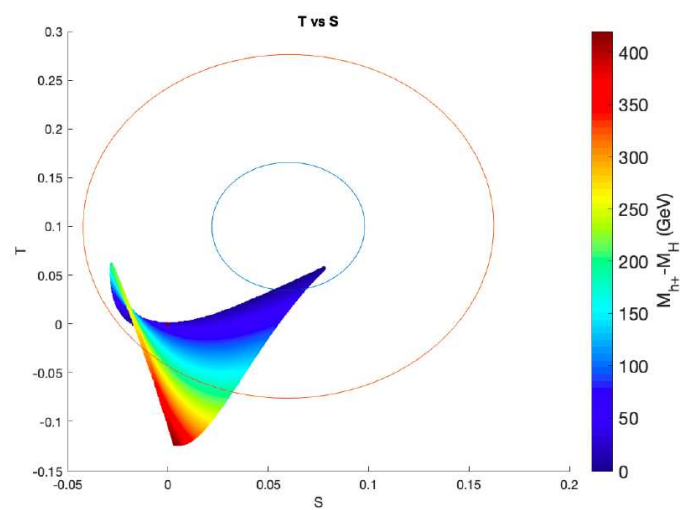

FIGURE 3. Effect of the S-T Constraint on the $M_{h+}-M_{H}$ mass split. Figure shows the color map of the $M_{h+}-M_{H}$ mass split in the S-T plane together with the $1-\sigma$ (blue ellipse) and $2 \sigma \chi^{2}$ (red ellipse) confidence intervals calculated with two degrees of freedom.

cision Tests (EWPT), we will express the EWPT bounds in terms of three measurable quantities, called $\mathrm{S}$, $\mathrm{T}$, and $\mathrm{U}$, that parameterize contributions from BSM physics to Electroweak radiative corrections [30]. The contribution to the $\mathrm{S}$ and $\mathrm{T}$ parameters [25] in this framework can be written as

$$
\begin{aligned}
S & =\frac{1}{72 \pi\left(x_{2}^{2}-x_{1}^{2}\right)^{3}}\left[x_{2}^{6} f_{a}\left(x_{2}\right)\right. \\
& -x_{1}^{6} f_{a}\left(x_{1}\right)+9 x_{2}^{2} x_{1}^{2}\left(x_{2}^{2} f_{b}\left(x_{2}\right)\right. \\
& \left.\left.-x_{1}^{2} f_{b}\left(x_{1}\right)\right)\right]
\end{aligned}
$$

where,

$$
\begin{array}{r}
x_{1}=\frac{m_{h}}{m_{h+}}, x_{2}=\frac{m_{H}}{m_{h+}}, f_{a}=-5+12 \log (x), \\
f_{b}=3-4 \log (x)
\end{array}
$$

and

$$
\begin{aligned}
T & =\frac{1}{32 \pi^{2} \alpha v^{2}} \\
& *\left[f_{c}\left(m_{h+}^{2}, m_{H}^{2}\right)+f_{c}\left(m_{h+}^{2}, m_{h}^{2}\right)-f_{c}\left(m_{H}^{2}, m_{h}^{2}\right)\right]
\end{aligned}
$$

where,

$$
\begin{array}{r}
f_{c}=\frac{x+y}{2}-\frac{x y}{x-y} \log \left(\frac{x}{y}\right), x \neq y \\
f_{c}=0, x=y
\end{array}
$$

With $\mathrm{U}$ fixed to be zero, the central values of $\mathrm{S}$ and $\mathrm{T}$, assuming a SM Higgs boson mass of $m_{h}=125 \mathrm{GeV}$, are given by [31]:

$$
S=0.06 \pm 0.09, T=0.1 \pm 0.07
$$

In Figure 3, we can see the allowed parameter space in this framework with reference to the Electroweak precision bounds (S, T). The Figure 3 represents the color map 
of the $\left(m_{h+}-m_{H}\right)$ mass split in the (S; T) plane together with the $1-\sigma$ and $2 \sigma \chi^{2}$ confidence intervals calculated with two degrees of freedom. One can see that EWPT data prefer a modest $\left(m_{h+}-m_{H}\right)$ mass split below about $100 \mathrm{GeV}$, which is due to the roles and respective range of variation for the following $\mathrm{S}$ and $\mathrm{T}$ parameter bound. It should be noted that in this framework the scalar $(H)$ and the pseudo-scalar $(A)$ can be taken to be on the same footing. We have assumed this interchangeability in the analysis and have only accepted the physically realizable solutions. Additionally, we have taken the absolute value in the difference on the $\mathrm{z}$-axis. The dark matter candidate $H$ can also be produced through the unsuppressed 4-point coupling $\mathrm{ZZHH}$ as seen in Eqn(18). We consider the decay process $Z \rightarrow Z^{*} H H \rightarrow f f H H$. The decay width with an assumption of $m_{H}=0$ gives [32]

$$
\sum_{f} \Gamma(Z \rightarrow f \bar{f} H H) \simeq 2.5 \times 10^{-7} \mathrm{GeV}
$$

The charged scalar particle $H^{ \pm}$can be produced via DrellYan process and it will be quite elusive to observe at the LHC. As the mass of the heavier charged scalar $m_{H}^{ \pm}$is greater than the mass of the neutral scalar $m_{H}, H^{ \pm}$can decay to $H^{ \pm} \rightarrow W^{ \pm} H$. It will be quite elusive to discover $H^{ \pm}$with the decay products of " $2 \mathrm{~W}$-boson + Miss $E_{T}$ " final states at the LHC. If the mass of the pseudoscalar $A$ is less than the mass of the charged scalar $H^{ \pm}, H^{ \pm}$can also decay to $H^{ \pm} \rightarrow W^{ \pm} A$. On the other hand, the pseudoscalar $A$ (depending on the actual mass hierarchy) can also decay to the charged scalar $H^{ \pm}$in association with a $W^{ \pm}$if $m_{A}>m_{H}^{ \pm}$. In the case of $m_{H^{ \pm}}>m_{w}+m_{H / A}$, the expressions for the partial decay widths of the heavy charged scalar $H^{ \pm}$are given by,

$$
\begin{aligned}
\Gamma\left(H^{ \pm} \rightarrow W^{ \pm} H / A\right) & =\frac{g^{2}}{64 \pi M_{w}^{2} M_{H^{ \pm}}^{3}} \\
& *\left(\left(M_{H^{ \pm}}^{2}-M_{H / A}^{2}-M_{w}^{2}\right)^{2}-4 M_{H / A}^{2} M_{w}^{2}\right)^{3 / 2}
\end{aligned}
$$

Recent ATLAS searches [33] performed for the search for chargino direct-production has found that In the scenario of $\tilde{\chi}_{1}^{+} \tilde{\chi}_{1}^{-}$pair production with $\tilde{\chi}_{1}^{ \pm}$decaying into and a $\tilde{\chi}_{1}^{0}$ and a W boson, $\tilde{\chi}_{1}^{ \pm}$masses in the ranges $100^{\sim} 105 \mathrm{GeV}$, $120^{\smile} 135 \mathrm{GeV}$ and $145^{\smile} 160 \mathrm{GeV}$ are excluded at $95 \% \mathrm{CL}$ for a massless $\tilde{\chi}_{1}^{0}$.

\section{SUMMARY AND CONCLUSIONS}

We have presented a simple twist in the well studied two Higgs doublet model in the form of adding an interchange symmetry to the two Higgs doublets $\left(\Phi_{1} \leftrightarrow\right.$ $\left.\Phi_{2}\right)$. A residual $Z_{2}$ symmetry that remains unbroken after the original symmetry $\Phi_{1} \leftrightarrow \Phi_{2}$ is spontaneously broken making the charged scalars $H^{ \pm}$, the neutral scalar $H$ and the pseudoscalar $A$ to have $Z_{2}$ negative charges and the other fields remaining $Z_{2}$ positive. This in turn makes the lightest $Z_{2}$ negative neutral scalar $H$ to be the DM candidate. This neutral scalar $H$ can be much lighter in mass than the Standard Model-(SM) like neutral scalar $h$ $\left(m_{h} \simeq 125 \mathrm{GeV}\right)$. Interestingly this lighter neutral scalar $H$ as well as the charged scalars $H^{ \pm}$and the pseudoscalar $A$ do not couple to the fermions. The lighter neutral scalars also don't have the usual three point couplings with the Gauge bosons $\left(W^{ \pm}\right.$and $\left.Z\right)$ present in the Standard Model; however has four-point couplings with $W^{ \pm}$and $Z$. The only channel to produce the dark matter $H$ is through the decays of the SM-like $h$ having an extra invisible decay channel through $h \rightarrow H H$. The Invisible decay branching ratio can be as large as $B r_{i n v h}<26 \%$. We study the parameter space of the effective coupling $\lambda^{*}$ between the neutral scalars $(h H H)$ and the mass of the dark matter $m_{H}$. We also comment on the Electroweak constraints in this scenario and also the other possible phenomenology for the charged scalars $H^{ \pm}$and pseudoscalar $A$ and the dark matter experimental bounds.

\section{ACKNOWLEDGMENTS}

SC is supported by the Holy Cross Bachelor Ford Summer fellowship '20-'21 and would like to thank S. Nandi for useful discussions, Colby College, Fermilab and College of the Holy Cross for their hospitality where part of this work was completed. H. B would like to thank Colby College and Yale University for providing the computational resources.

\section{EDITOR'S NOTE}

This manuscript was submitted to the Association of Nepali Physicists in America (ANPA) Conference 2021 for publication in the special issue of Journal of Nepal Physical Society.

\section{REFERENCES}

1. G. Aad et al., Phys. Lett. B 716, 1-29 (2012)

2. S. Chatrchyan et al., Phys. Lett. B 716, 30-61 (2012).

3. T. D. Lee, Phys. Rev. D 8, 1226-1239 (1973).

4. J. Gunion, H. Haber, G. Kane, and S. Dawson, Vol. 80 (2000).

5. A. Djouadi, Phys. Rept. 459, 1-241 (2008).

6. G. C. Branco, P. M. Ferreira, L. Lavoura, M. N. Rebelo, M. Sher, and J. P. Silva, Phys. Rept. 516, 1-102 (2012).

7. U. Maitra, B. Mukhopadhyaya, S. Nandi, S. K. Rai, and A. Shivaji, Phys. Rev. D 89, 055024 (2014).

8. W. Altmannshofer, S. Gori, and G. D. Kribs, Phys. Rev. D 86, 115009 (2012)

9. S. Chang, S. K. Kang, J.-P. Lee, K. Y. Lee, S. C. Park, and J. Song, JHEP 05, 075 (2013).

10. C.-Y. Chen and S. Dawson, Phys. Rev. D 87, 055016 (2013).

11. A. Celis, V. Ilisie, and A. Pich, JHEP 07, 053 (2013).

12. B. Grinstein and P. Uttayarat, JHEP 06, 094 (2013).

13. B. Coleppa, F. Kling, and S. Su, JHEP 01, 161 (2014).

14. C.-Y. Chen, S. Dawson, and M. Sher, Phys. Rev. D 88, 015018 (2013).

15. O. Eberhardt, U. Nierste, and M. Wiebusch, JHEP 07, 118 (2013)

16. N. Craig, J. Galloway, and S. Thomas, (2013). 
17. L. Maiani, A. D. Polosa, and V. Riquer, Phys. Lett. B 724, 274-277 (2013).

18. L. Wang and X.-F. Han, JHEP 04, 128 (2014).

19. J. Baglio, O. Eberhardt, U. Nierste, and M. Wiebusch, Phys. Rev. D 90, 015008 (2014).

20. S. Kanemura, H. Yokoya, and Y.-J. Zheng, Nucl. Phys. B 886, 524-553 (2014).

21. B. Dumont, J. F. Gunion, Y. Jiang, and S. Kraml, Phys. Rev. D 90, 035021 (2014).

22. G. Aarons et al. (ILC), (2007), arXiv:0709.1893 [hep-ph].

23. J. Beringer et al., Phys. Rev. D 86, 010001 (2012).

24. P. A. R. Ade et al., Astron. Astrophys. 571, A16 (2014).

25. A. Hook, E. Izaguirre, and J. G. Wacker, Adv. High Energy Phys. 2011, 859762 (2011)

26. E. Aprile et al., Phys. Rev. Lett. 109, 181301 (2012).

27. G. Elor, N. L. Rodd, T. R. Slatyer, and W. Xue, JCAP 06, 024 (2016).

28. M. Aaboud et al., Phys. Rev. Lett. 122, 231801 (2019).

29. (2014).

30. M. E. Peskin and T. Takeuchi, Phys. Rev. D 46, 381-409 (1992).

31. M. Baak, J. Cúth, J. Haller, A. Hoecker, R. Kogler, K. Mönig, M. Schott, and J. Stelzer, Eur. Phys. J. C 74, 3046 (2014).

32. C. Patrignani et al., Chin. Phys. C 40, 100001 (2016).

33. G. Aad et al., JHEP 05, 071 (2014) 